\title{
A Practical-Time Related-Key Attack on the KASUMI Cryptosystem Used in GSM and 3G Telephony
}

\author{
Orr Dunkelman, Nathan Keller*, and Adi Shamir \\ Faculty of Mathematics and Computer Science \\ Weizmann Institute of Science \\ P.O. Box 26, Rehovot 76100, Israel \\ \{orr.dunkelman, nathan.keller, adi.shamir\}@weizmann.ac.il
}

\begin{abstract}
The privacy of most GSM phone conversations is currently protected by the $20+$ years old A5/1 and A5/2 stream ciphers, which were repeatedly shown to be cryptographically weak. They will soon be replaced by the new A5/3 (and the soon to be announced A5/4) algorithm based on the block cipher KASUMI, which is a modified version of MISTY. In this paper we describe a new type of attack called a sandwich attack, and use it to construct a simple distinguisher for 7 of the 8 rounds of KASUMI with an amazingly high probability of $2^{-14}$. By using this distinguisher and analyzing the single remaining round, we can derive the complete 128 bit key of the full KASUMI by using only 4 related keys, $2^{26}$ data, $2^{30}$ bytes of memory, and $2^{32}$ time. These complexities are so small that we have actually simulated the attack in less than two hours on a single PC, and experimentally verified its correctness and complexity. Interestingly, neither our technique nor any other published attack can break MISTY in less than the $2^{128}$ complexity of exhaustive search, which indicates that the changes made by ETSI's SAGE group in moving from MISTY to KASUMI resulted in a much weaker cipher.
\end{abstract}

\section{Introduction}

The privacy and security of GSM cellular telephony is protected by the A5 family of cryptosystems. The first two members of this family, A5/1 (developed primarily for European markets) and A5/2 (developed primarily for export markets) were designed in the late 1980's in an opaque process and were kept secret until they were reverse engineered in 1999 from actual handsets [14]. Once published, it became clear that A5/2 provided almost no security, and A5/1 could be attacked with practical complexity by a variety of techniques (e.g., [21216]). The most recent attack was announced in December 2009, when a team of cryptographers led by Karsten Nohl [1] published a 2 terabyte rainbow table for A5/1, which makes it easy to derive the session key of any particular conversation with minimal hardware support.

\footnotetext{
* The second author was partially supported by the Koshland center for basic research.
} 
In response to these developments, the GSM Association had stated in [26] that they might speed up their transition to a new cryptosystem called A $5 / 3$, and they plan to discuss this matter in a meeting that was held in February 2010. This algorithm was developed for GSM telephony in 2002, and its specifications were published in 2003 [24. It is already implemented in about $40 \%$ of the three billion available handsets, but very few of the 800 mobile carriers in more than 200 countries which currently use GSM cellular telephony have switched so far to the new standard. Once adopted, A5/3 will become one of the most widely used cryptosystems in the world, and its security will become one of the most important practical issues in cryptography.

The core of the A5/3 cryptosystem, as well as of the UAE1 cryptosystem (which replaces A5/3 in the third generation telephony networks), is the KASUMI block cipher, which is based on the MISTY block cipher which was published at FSE 1997 by Matsui [22]. MISTY has 64-bit blocks, 128-bit keys, and a complex recursive Feistel structure with 8 rounds, each one of which consists of 3 rounds, each one of which has 3 rounds of nonlinear SBox operations. MISTY has provable security properties against various types of attacks, and no attack is known on its full version. The best published attack can be applied to a 6 -round reduced variant of the 8-round MISTY, and has a completely impractical time complexity of more than $2^{123}$ [15]. However, the designers of A5/3 decided to make MISTY faster and more hardware-friendly by simplifying its key schedule and modifying some of its components. In 25, the designers provide a rationale for each one of these changes, and in particular they analyze the resistance of KASUMI against related-key attacks by stating that "removing all the FI functions in the key scheduling part makes the hardware smaller and/or reduces the key set-up time. We expect that related key attacks do not work for this structure". The best attack found by the designers and external evaluators of KASUMI is described as follows:

"There are chosen plaintext and/or related-key attacks against KASUMI reduced to 5 rounds. We believe that with further analysis it might be possible to extend some attacks to 6 rounds, but not to the full 8 round KASUMI."

The existence of better related-key attacks on the full KASUMI was already shown in 821 . Their attack had a data complexity of $2^{54.6}$ and time complexity of $2^{76.1}$, which are impractical but better than exhaustive search. In this paper we develop a new attack, which requires only 4 related keys, $2^{26}$ data, $2^{30}$ bytes of memory, and $2^{32}$ time. Since these complexities are so low, we could verify our attack experimentally, and our unoptimized implementation on a single $\mathrm{PC}$ recovered about 96 key bits in a few minutes, and the complete 128 bit key in less than two hours. Careful analysis of our attack technique indicates that it can not be applied against the original MISTY, since it exploits a sequence of coincidences and lucky strikes which were created when MISTY was changed to KASUMI by ETSI's SAGE group. This calls into question both the design of KASUMI and its security evaluation against related-key attacks. However, we would like to emphasize that even though our attack on the underlying 
cryptosystem has a practical time complexity, we do not claim that we can practically apply such a related key attack to the way KASUMI is used in the f8 and f9 modes of operation in cellular telephony.

We use a new type of attack which is an improved version of the boomerang attack introduced in [27]. We call it a "sandwich attack", since it uses a distinguisher which is divided into three parts: A thick slice ("bread") at the top, a thin slice ("meat") in the middle, and a thick slice ("bread") at the bottom. The top and bottom parts are assumed to have high probability differential characteristics, which can be combined into consistent quartet structures by the standard boomerang technique. However, in our case they are separated by the additional middle slice, which can significantly reduce the probability of the resulting boomerang structure. Nevertheless, as we show in this paper, careful analysis of the dependence between the top and bottom differentials allows us in some cases to combine the two properties above and below the middle slice with an enhanced probability. In particular, we show that in the case of KASUMI we can use top and bottom 3-round differential characteristics with an extremely high probability of $2^{-2}$ each, and combine them via a middle 1-round slice in such a way that the "price in probability" of the combination is $2^{-6}$, instead of the $2^{-32}$ we would expect from a naive analysis. This increases the probability of our 7 -round distinguisher from $2^{-40}$ to $2^{-14}$, and has an even bigger impact on the amount of data and the time complexity of the attack due to the quadratic dependence of the number of cases we have to sample on the distinguishing probability. Such a three level structure was used in several previous attacks such as [1011] (where it was called the "Feistel switch" or the "middle round S-box trick"), but to the best of our knowledge it was always used in the past in simpler situations in which the transition probability through the middle layer (in at least one direction) was 1 due to the structural properties of a single Feistel round, or due to the particular construction of a given SBox. Our sandwich attack is the first nontrivial application of such a structure, and the delicacy of the required probabilistic analysis is demonstrated by the fact that a tiny change in the key schedule of KASUMI (which has no effect on the differential probabilities of the top and bottom layers) can change the probability of the transition in the middle of the distinguisher from the surprisingly high value of $2^{-6}$ to 0 .

This paper is organized as follows: Section 2 describes the new sandwich attack, and discusses the transition between the top and bottom parts of the cipher through the middle slice of the sandwich. Section 3 describes the KASUMI block cipher. Section 4 describes our new 7-round distinguisher for KASUMI which has a probability of $2^{-14}$. In Section 5 we use the new distinguisher to develop a practical-time key recovery attack on the full KASUMI cryptosystem.

\section{Sandwich Attacks}

In this section we describe the technique used in our attacks on KASUMI. We start with a description of the basic (related-key) boomerang attack, and then 
we describe a new framework, which we call a (related-key) sandwich attack, that exploits the dependence between the underlying differentials to obtain a more accurate estimation of the probability of the distinguisher. We note that the idea of using dependence between the differentials in order to improve the boomerang distinguisher was implicitly proposed by Wagner [27, and was also used in some simple scenarios in 10[11]. Therefore, our framework can be considered as a formal treatment and generalization of the ideas proposed in [10]11[27].

\subsection{The Basic Related-Key Boomerang Attack}

The related-key boomerang attack was introduced by Kim et al. 20]18, and independently by Biham et al. [7], as a combination of the boomerang attack [27. and the related-key differential attack [19. In this attack, the cipher is treated as a cascade of two sub-ciphers $E=E_{1} \circ E_{0}$, and related-key differentials of $E_{0}$ and $E_{1}$ are combined into an adaptive chosen plaintext and ciphertext distinguisher for $E$.

Let us assume that there exists a related-key differential $\alpha \rightarrow \beta$ for $E_{0}$ under key difference $\Delta K_{a b}$ with probability $p$. (i.e., $\operatorname{Pr}\left[E_{0(K)}(P) \oplus E_{0\left(K \oplus K_{a b}\right)}(P \oplus \alpha)=\right.$ $\beta]=p$, where $E_{0(K)}$ denotes encryption through $E_{0}$ under the key $\left.K\right)$. Similarly, we assume that there exists a related-key differential $\gamma \rightarrow \delta$ for $E_{1}$ under key difference $\Delta K_{a c}$ with probability $q$. The related-key boomerang distinguisher requires encryption/decryption under the secret key $K_{a}$, and under the relatedkeys $K_{b}=K_{a} \oplus \Delta K_{a b}, K_{c}=K_{a} \oplus \Delta K_{a c}$, and $K_{d}=K_{c} \oplus \Delta K_{a b}=K_{b} \oplus \Delta K_{a c}$.

A boomerang quartet is generated by picking a plaintext $P_{a}$ at random, and asking for its encryption under $K_{a}$, namely, $C_{a}=E_{K_{a}}\left(P_{a}\right)$. Then, $P_{b}=P_{a} \oplus \alpha$ is encrypted under $K_{b}$ to obtain $C_{b}=E_{K_{b}}\left(P_{b}\right)$. Two new ciphertexts are computed, $C_{c}=C_{a} \oplus \delta$ and $C_{d}=C_{b} \oplus \delta$. Then, $C_{c}$ is decrypted under $K_{c}$, and $C_{d}$ is decrypted under $K_{d}$, i.e., $P_{c}=E_{K_{c}}^{-1}\left(C_{c}\right)$ and $P_{d}=E_{K_{d}}^{-1}\left(C_{d}\right)$. If $P_{c} \oplus P_{d}=\alpha$, a right boomerang quartet is found. The left side of Figure 1 describes such a right related-key boomerang quartet.

For a random permutation the probability that the last condition is satisfied is $2^{-n}$, where $n$ is the block size. For $E$, the probability that the pair $\left(P_{a}, P_{b}\right)$ is a right pair with respect to the first differential (i.e., the probability that the intermediate difference after $E_{0}$ equals $\beta$ ) is $p$. Assuming independence, the probability that both pairs $\left(C_{a}, C_{c}\right)$ and $\left(C_{b}, C_{d}\right)$ are right pairs with respect to the second differential is $q^{2}$. If all these are right pairs, then $E_{1}^{-1}\left(C_{c}\right) \oplus E_{1}^{-1}\left(C_{d}\right)=$ $\beta=E_{0}\left(P_{c}\right) \oplus E_{0}\left(P_{d}\right)$. Thus, with probability $p, P_{c} \oplus P_{d}=\alpha$. Hence, the total probability of this quartet of plaintexts and ciphertexts to satisfy the condition $P_{c} \oplus P_{d}=\alpha$ is at least $(p q)^{2}$. Therefore, if $p q \gg 2^{-n / 2}$, the algorithm above allows to distinguish $E$ from a random permutation given $O\left((p q)^{-2}\right)$ adaptively chosen plaintexts and ciphertexts.

The distinguisher can be improved by considering multiple differentials of the form $\alpha \rightarrow \beta^{\prime}$ and $\gamma^{\prime} \rightarrow \delta$ (for the same $\alpha$ and $\delta$ ). We omit this improvement here since it is not used in our attack on KASUMI, and refer the reader to [7]. For 
a rigorous treatment of the related-key boomerang attack, including a discussion of the independence assumptions the attack relies upon, we refer the interested reader to 2123 .

\subsection{Related-Key Sandwich Attacks}

In this framework we consider the cipher as a cascade of three sub-ciphers: $E=E_{1} \circ M \circ E_{0}$. Our assumptions are the same as in the basic attack: We assume that there exists a related-key differential $\alpha \rightarrow \beta$ for $E_{0}$ under key difference $\Delta K_{a b}$ with probability $p$, and a related-key differential $\gamma \rightarrow \delta$ for $E_{1}$ under key difference $\Delta K_{a c}$ with probability $q$. The attack algorithm is also exactly the same as in the basic attack (ignoring the middle sub-cipher $M$ ). However, the analysis is more delicate and requires great care in analyzing the dependence between the various distributions.

The main idea behind the sandwich attack is the transition in the middle. In the basic boomerang attack, if the pair $\left(P_{a}, P_{b}\right)$ is a right pair with respect to the first differential, and both pairs $\left(C_{a}, C_{c}\right)$ and $\left(C_{b}, C_{d}\right)$ are right pairs with respect to the second differential, then we have

$$
\left(X_{a} \oplus X_{b}=\beta\right) \wedge\left(X_{a} \oplus X_{c}=\gamma\right) \wedge\left(X_{b} \oplus X_{d}=\gamma\right)
$$

where $X_{i}$ is the intermediate encryption value of $P_{i}$, and thus

$$
X_{c} \oplus X_{d}=\left(X_{c} \oplus X_{a}\right) \oplus\left(X_{a} \oplus X_{b}\right) \oplus\left(X_{b} \oplus X_{d}\right)=\beta \oplus \gamma \oplus \gamma=\beta,
$$

resulting in $P_{c} \oplus P_{d}=\alpha$ with probability $p$ (see Figure 1 ).

In the new sandwich framework, instead of condition (1), we get

$$
\left(X_{a} \oplus X_{b}=\beta\right) \wedge\left(Y_{a} \oplus Y_{c}=\gamma\right) \wedge\left(Y_{b} \oplus Y_{d}=\gamma\right)
$$

Therefore, the probability of the three-layer related-key boomerang distinguisher is $p^{2} q^{2} r$, where

$$
r=\operatorname{Pr}\left[\left(X_{c} \oplus X_{d}=\beta\right) \mid\left(X_{a} \oplus X_{b}=\beta\right) \wedge\left(Y_{a} \oplus Y_{c}=\gamma\right) \wedge\left(Y_{b} \oplus Y_{d}=\gamma\right)\right] .
$$

Without further assumptions on $M, r$ is expected to be very low (close to $2^{-n}$ ), and thus the distinguisher is expected to fail. However, as observed in [10 1127, in some cases the differentials in $E_{0}$ and $E_{1}$ can be chosen such that the probability penalty $r$ in going through the middle sub-cipher (in at least one direction) is 1 , which is much higher than expected.

An example of this phenomenon, introduced in [27] and described in [11. under the name "Feistel switch", is the following. Let $E$ be a Feistel cipher, decomposed as $E=E_{1} \circ M \circ E_{0}$, where $M$ consists of one Feistel round (see Figure 2). Assume that the differentials $\alpha \rightarrow \beta$ (for $E_{0}$ ) and $\gamma \rightarrow \delta$ (for $E_{1}$ ) have no key difference (i.e., $\Delta K_{a b}=\Delta K_{a c}=0$ ), and satisfy $\beta^{R}=\gamma^{L}$ (i.e., the right half of $\beta$ equals the left half of $\gamma$ ). We would like to compute the value of $r$. 


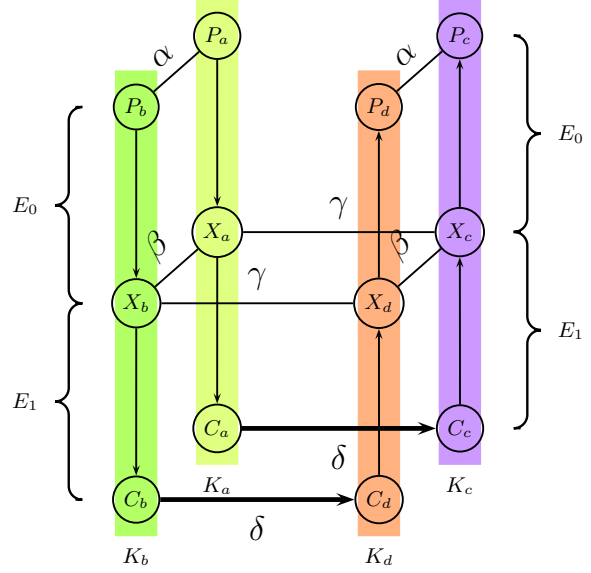

A Related-Key Boomerang Quartet

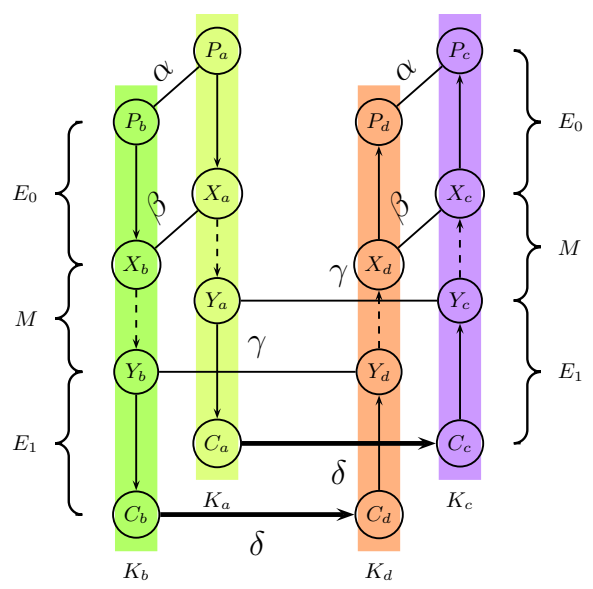

A Related-Key Sandwich Quartet

Fig. 1. Related-Key Boomerang and Sandwich Quartets

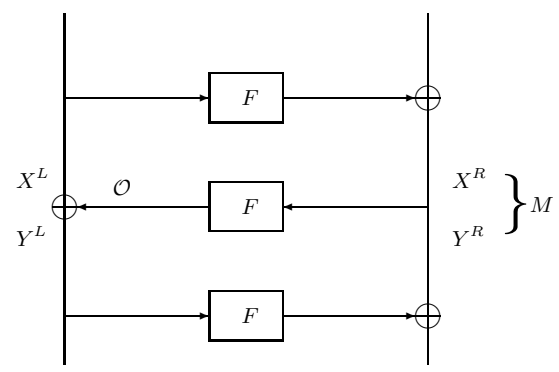

Fig. 2. A Feistel construction. $M$ is the second round

Assume that condition (3) holds. In this case, by the Feistel construction, $X_{i}^{R}=Y_{i}^{L}$ for all $i$, we have

$$
X_{a}^{R} \oplus X_{b}^{R}=\beta^{R}=\gamma^{L}=X_{a}^{R} \oplus X_{c}^{R}=X_{b}^{R} \oplus X_{d}^{R},
$$

and thus,

$$
\left(X_{a}^{R}=X_{d}^{R}\right) \quad \text { and } \quad\left(X_{b}^{R}=X_{c}^{R}\right) .
$$

Therefore, the output values of the F-function in the Feistel round represented by $M$, denoted by $\left(\mathcal{O}_{a}, \mathcal{O}_{b}, \mathcal{O}_{c}, \mathcal{O}_{d}\right)$, satisfy

$$
\left(\mathcal{O}_{a}=\mathcal{O}_{d}\right) \quad \text { and } \quad\left(\mathcal{O}_{b}=\mathcal{O}_{c}\right) .
$$

Since by the Feistel construction, $X_{i}^{L}=Y_{i}^{R} \oplus \mathcal{O}_{i}$ and by condition (3),$Y_{a} \oplus Y_{b} \oplus$ $Y_{c} \oplus Y_{d}=0$, it follows that

$$
X_{a} \oplus X_{b} \oplus X_{c} \oplus X_{d}=0,
$$

which by condition (3) implies $X_{c} \oplus X_{d}=\beta$. Thus, in this case we get 


$$
r=\operatorname{Pr}\left[\left(X_{c} \oplus X_{d}=\beta\right) \mid\left(X_{a} \oplus X_{b}=\beta\right) \wedge\left(Y_{a} \oplus Y_{c}=\gamma\right) \wedge\left(Y_{b} \oplus Y_{d}=\gamma\right)\right]=1,
$$

independently of the choice of the F-function used.

Other examples of the same phenomenon are considered in [10] (under the name "middle round S-box trick"), and in [1] (under the names "ladder switch" and "S-box switch"). All these examples are methods for $r=1$.

Our attack on KASUMI is the first non-trivial example of this phenomenon in which a careful analysis shows that $r$ is smaller than 1 , but much larger than its expected value under the standard independence assumptions. In our attack, the cipher $E$ (7-round KASUMI) is a Feistel construction, $M$ consists of a single round, and $\beta=\gamma$. However, the argument presented above cannot be applied directly since there is a non-zero key difference in $M$, and thus a zero input difference to the F-function does not imply zero output difference. Instead, we analyze the F-function thoroughly and show that in this case, $r=2^{-6}$ (instead of $2^{-32}$, which is the expected value for a random Feistel round in a 64-bit cipher).

Remark 1. We note that our treatment of the sandwich distinguisher allows us to specify the precise independence assumptions we rely upon. Since $r$ is defined as a conditional probability, the only independence assumptions we use are between the differentials of $E_{0}$ and $E_{1}$, and thus the formula $p^{2} q^{2} r$ relies on exactly the same assumptions as the ordinary boomerang attack. Moreover, in our case the assumptions seem more likely to hold since the insertion of $M$ in the middle decreases the potential dependencies between the differentials for $E_{0}$ and the differentials for $E_{1}$. In [101127], this situation was treated as a "trick" allowing to increase the probability of the distinguisher, or in other words, as a failure of the formula $p^{2} q^{2}$ in favor of the attacker. This approach is problematic since once we claim that the entire formula does not hold due to dependencies, we cannot rely on independence assumptions in other places where such dependencies were not found yet.

\section{The KASUMI Block Cipher}

KASUMI 24] is a 64-bit block cipher with 128-bit keys. It has a recursive Feistel structure, following its ancestor MISTY. The cipher has eight Feistel rounds, where each round is composed of two functions: the $F O$ function which is in itself a 3-round 32-bit Feistel construction, and the $F L$ function that mixes a 32-bit subkey with the data in a linear way. The order of the two functions depends on the round number: in the even rounds the $F O$ function is applied first, and in the odd rounds the $F L$ function is applied first.

The $F O$ function also has a recursive structure: its $F$-function, called $F I$, is a four-round Feistel construction. The $F I$ function uses two non-linear S-boxes $S 7$ and $S 9$ (where $S 7$ is a 7 -bit to 7 -bit permutation and $S 9$ is a 9 -bit to 9 -bit permutation), and accepts an additional 16-bit subkey, which is mixed with the data. In total, a 96-bit subkey enters $F O$ in each round - 48 subkey bits are used in the $F I$ functions and 48 subkey bits are used in the key mixing stages. 

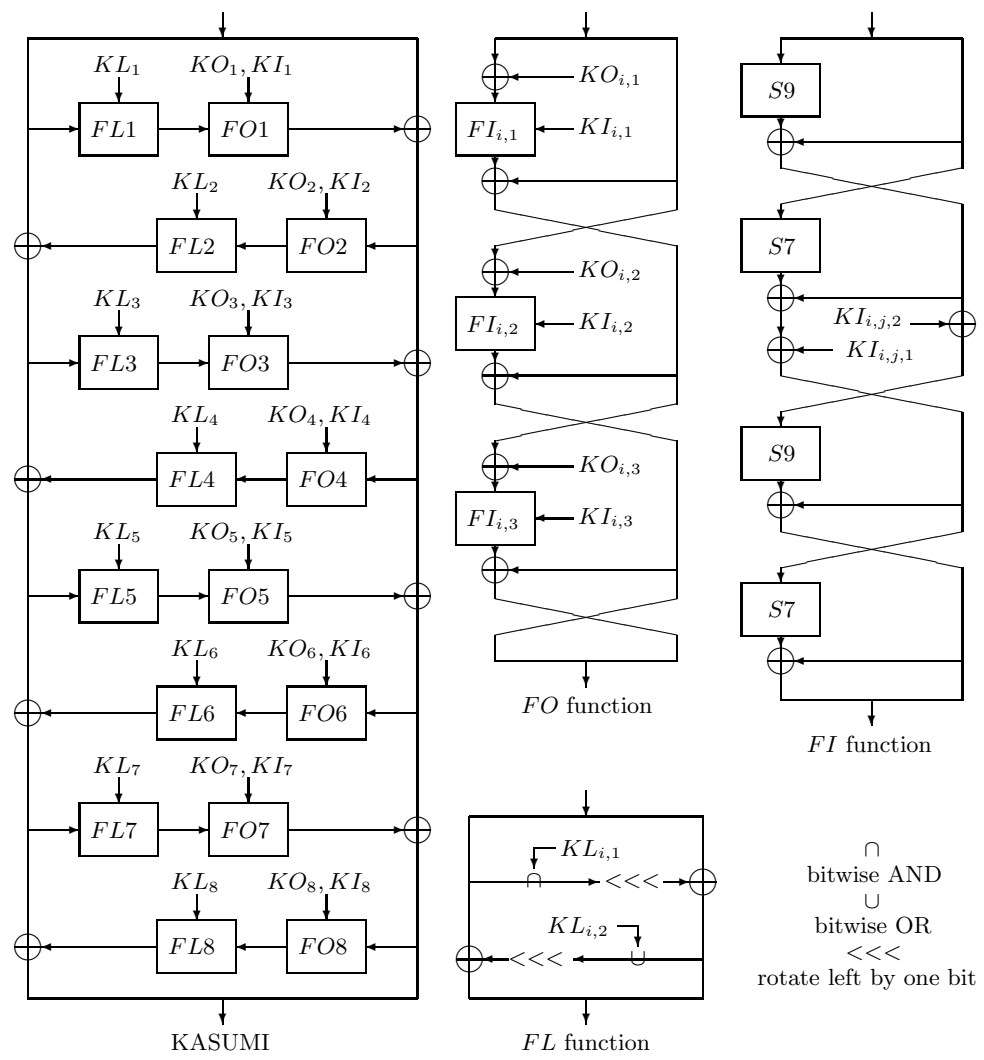

Fig. 3. Outline of KASUMI

Table 1. KASUMI's Key Schedule Algorithm

\begin{tabular}{|c|c|c|c|c|c|c|c|}
\hline Round & $K L_{i, 1}$ & $K L_{i, 2}$ & $K O_{i, 1}$ & $K O_{i, 3}$ & $K I_{i}$, & $K I_{i, 2}$ & $K I_{i, 3}$ \\
\hline 1 & $K_{1} \lll 1$ & $K_{3}^{\prime}$ & $K_{2} \lll$ & $K_{6} \lll 8 K_{7} \lll 13$ & $K_{5}^{\prime}$ & $K_{4}^{\prime}$ & $K_{8}^{\prime}$ \\
\hline 2 & $K_{2} \lll 1$ & $K_{4}^{\prime}$ & $K_{3} \lll$ & $K_{7} \lll 8 K_{8} \lll 13$ & $K_{6}^{\prime}$ & $K_{5}^{\prime}$ & $K_{1}^{\prime}$ \\
\hline 3 & $K_{3} \lll 1$ & $K_{5}^{\prime}$ & $K_{4} \lll 5$ & $K_{8} \lll 8 K_{1} \lll 13$ & $K_{7}^{\prime}$ & $K_{6}^{\prime}$ & $K_{2}^{\prime}$ \\
\hline 4 & $K_{4} \lll 1$ & $K_{6}^{\prime}$ & $K_{5} \lll 5$ & $K_{1} \lll 8 K_{2} \lll 13$ & $K_{8}^{\prime}$ & $K_{7}^{\prime}$ & $K_{3}^{\prime}$ \\
\hline 5 & $K_{5} \lll 1$ & $K_{7}^{\prime}$ & $K_{6} \lll 5$ & $K_{2} \lll 8 K_{3} \lll 13$ & $K_{1}^{\prime}$ & $K_{8}^{\prime}$ & $K_{4}^{\prime}$ \\
\hline 6 & $K_{6} \lll 1$ & $K_{8}^{\prime}$ & $K_{7} \lll$ & $K_{3} \lll 8 K_{4} \lll 13$ & $K_{2}^{\prime}$ & $K_{1}^{\prime}$ & $K_{5}^{\prime}$ \\
\hline 7 & $K_{7} \lll 1$ & $K_{1}^{\prime}$ & $K_{8} \lll$ & $K_{4} \lll 8 K_{5} \lll 13$ & $K_{3}^{\prime}$ & $K_{2}^{\prime}$ & $K_{6}^{\prime}$ \\
\hline 8 & $K_{8} \lll 1$ & $K_{2}^{\prime}$ & $K_{1} \lll 5$ & $K_{5} \lll 8 K_{6} \lll 13$ & $K_{4}^{\prime}$ & $K_{3}^{\prime}$ & $K_{7}^{\prime}$ \\
\hline
\end{tabular}

The FL function accepts a 32-bit input and two 16-bit subkey words. One subkey word affects the data using the OR operation, while the second one affects the data using the AND operation. We outline the structure of KASUMI and its parts in Fig. 3 . 
The key schedule of KASUMI is much simpler than the original key schedule of MISTY, and the subkeys are linearly derived from the key. The 128-bit key $K$ is divided into eight 16-bit words: $K_{1}, K_{2}, \ldots, K_{8}$. Each $K_{i}$ is used to compute $K_{i}^{\prime}=K_{i} \oplus C_{i}$, where the $C_{i}$ 's are fixed constants (we omit these from the paper, and refer the intrigued reader to [24]). In each round, eight words are used as the round subkey (up to some in-word rotations). Hence, each 128-bit round subkey is a linearly modified version of the secret key. We summarize the details of the key schedule of KASUMI in Table 1.

\section{A Related-Key Sandwich Distinguisher for 7-Round KASUMI}

\subsection{The New Distinguisher}

In our distinguisher, we treat rounds $1-7$ of KASUMI as a cascade $E=E_{1} \circ M \circ$ $E_{0}$, where $E_{0}$ consists of rounds $1-3, M$ consists of round 4 , and $E_{1}$ consists of rounds $5-7$. The related-key differential we use for $E_{0}$ is a slight modification of the differential characteristic presented in [13, in which

$$
\alpha=\left(0_{x}, 00100000_{x}\right) \rightarrow\left(0_{x}, 00100000_{x}\right)=\beta .
$$

The corresponding key difference is $\Delta K_{a b}=\left(0,0,8000_{x}, 0,0,0,0,0\right)$, i.e., only the third key word has the single bit difference $\Delta K_{3}=8000_{x}$. This related-key differential is depicted in Figure 4. The related-key differential we use for $E_{1}$ is the same differential shifted by four rounds, in which the data difference is the same, but the key difference is $\Delta K_{a c}=\left(0,0,0,0,0,0,8000_{x}, 0\right)$ (to handle the different subkeys used in these rounds).

As shown in [13, the probability of each one of of these 3-round differential characteristics is $1 / 4$. In order to find the probability of the related-key sandwich distinguisher, we have to compute the probability

$$
\operatorname{Pr}\left[\left(X_{c} \oplus X_{d}=\beta\right) \mid\left(X_{a} \oplus X_{b}=\beta\right) \wedge\left(Y_{a} \oplus Y_{c}=\gamma\right) \wedge\left(Y_{b} \oplus Y_{d}=\gamma\right)\right],
$$

where $\left(X_{a}, X_{b}, X_{c}, X_{d}\right)$ and $\left(Y_{a}, Y_{b}, Y_{c}, Y_{d}\right)$ are the intermediate values before and after the middle slice of the sandwich during the encryption/decryption of the quartet $\left(P_{a}, P_{b}, P_{c}, P_{d}\right)$ (see the right side of Figure 11). This computation, which is a bit complicated, spans the rest of this subsection.

Consider a quartet $\left(P_{a}, P_{b}, P_{c}, P_{d}\right)$ for which the condition

$$
\left(X_{a} \oplus X_{b}=\beta\right) \wedge\left(Y_{a} \oplus Y_{c}=\gamma\right) \wedge\left(Y_{b} \oplus Y_{d}=\gamma\right)
$$

is satisfied. As explained in Section 2, since $M$ is a single Feistel round, this implies that

$$
\left(X_{a}^{R}=X_{d}^{R}\right) \wedge\left(X_{b}^{R}=X_{c}^{R}\right),
$$

where $X_{i}^{R}$ denotes the right half of $X_{i}$ that enters the function FO4. Moreover, as the right quarter of the differences $\beta=\gamma$ is zero, we have 


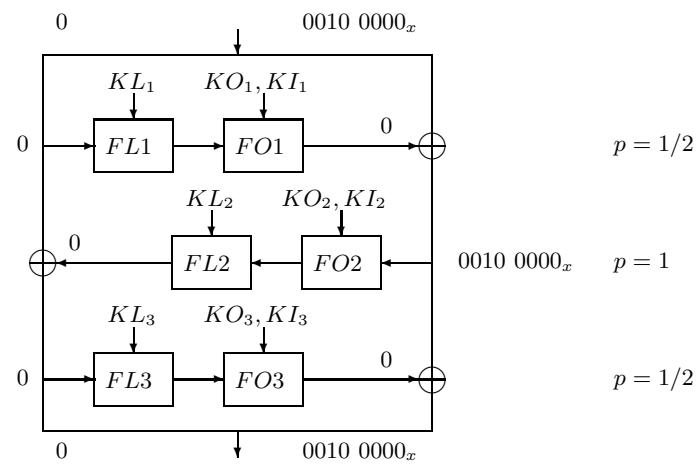

Fig. 4. 3-Round Related-Key Differential Characteristic of KASUMI

$$
X_{a}^{R R}=X_{b}^{R R}=X_{c}^{R R}=X_{d}^{R R},
$$

where $X_{i}^{R R}$ denotes the right half (i.e., the 16 right bits) of $X_{i}^{R}$.

Consider now the computation depicted in Figure 5 . The function FO4 is a 3 -round Feistel construction whose 32-bit values after round $j$ are denoted by $\left(X_{a}^{j}, X_{b}^{j}, X_{c}^{j}, X_{d}^{j}\right)$, and the function $F I$ is a 4 -round Feistel construction whose 16-bit values after round $j$ are denoted by $\left(I_{a}^{j}, I_{b}^{j}, I_{c}^{j}, I_{d}^{j}\right)$. Note that the key differences $\Delta K_{a b}$ and $\Delta K_{a c}$ affect in round 4 the subkeys $K I_{4,3}$ and $K I_{4,2}$, respectively, and in particular, there is no key difference in the first round of FO4. As a result, Equation (9) implies that

$$
\left(X_{a}^{1}=X_{d}^{1}\right) \wedge\left(X_{b}^{1}=X_{c}^{1}\right) .
$$

Furthermore, there is no key difference in the pairs corresponding to $\left(P_{a}, P_{b}\right)$ and $\left(P_{c}, P_{d}\right)$ in the second round of FO4, and thus Equation (10) implies

$$
\left(I_{a}^{2}=I_{b}^{2}\right) \wedge\left(I_{c}^{2}=I_{d}^{2}\right) .
$$

Combining equations (11) and (12), as depicted in Figure 5, we get the following relation in the right half of the intermediate values after round 3 of FO4:

$$
X_{a}^{3 R} \oplus X_{b}^{3 R} \oplus X_{c}^{3 R} \oplus X_{d}^{3 R}=0 .
$$

In the F-function of round 3 of FO4 we consider the pairs corresponding to $\left(P_{a}, P_{d}\right)$ and $\left(P_{b}, P_{c}\right)$. Since the key difference in these pairs (that equals $K_{a b} \oplus$ $\left.K_{a c}\right)$ affects only the subkey $K I_{4,3,1}$, Equation (11) implies

$$
I_{a}^{3 R} \oplus I_{b}^{3 R} \oplus I_{c}^{3 R} \oplus I_{d}^{3 R}=0
$$

in the right hand side of the output. In the left hand side of the output, the XOR of the four values is not necessarily equal to zero, due to the subkey difference that affects the inputs to the second $S 7$ in $F I_{4,3}$. However, if these 7-bit inputs, denoted by $\left(J_{a}, J_{b}, J_{c}, J_{d}\right)$, satisfy one of the conditions:

$$
\left(\left(J_{a}=J_{b}\right) \wedge\left(J_{c}=J_{d}\right)\right) \quad \text { or } \quad\left(\left(J_{a}=J_{c}\right) \wedge\left(J_{b}=J_{d}\right)\right),
$$


then Equation (14) implies

$$
I_{a}^{3 L} \oplus I_{b}^{3 L} \oplus I_{c}^{3 L} \oplus I_{d}^{3 L}=0 .
$$

Since we have $J_{a} \oplus J_{d}=J_{b} \oplus J_{c}$ (both are equal to the subkey difference in $K I_{4,3,1}$ ), each one of the two conditions in Equation (15) is expected to hold with probability $2^{-7}$. Therefore, combining Equations (13), (14), and (16) we get that the condition

$$
X_{a}^{3} \oplus X_{b}^{3} \oplus X_{c}^{3} \oplus X_{d}^{3}=0
$$

holds with probability $2^{-6}$.

Finally, since the $F L$ function is linear for a given key and there is no key difference in FL4, we can conclude that whenever Equation (17) holds, the outputs of the F-function in round 4 (denoted by $\left(O_{a}^{4}, O_{b}^{4}, O_{c}^{4}, O_{d}^{4}\right)$ ) satisfy

$$
O_{a}^{4} \oplus O_{b}^{4} \oplus O_{c}^{4} \oplus O_{d}^{4}=0
$$

with probability $2^{-6}$. Since by condition (요),

$$
Y_{a}^{L} \oplus Y_{b}^{L} \oplus Y_{c}^{L} \oplus Y_{d}^{L}=0,
$$

it follows that

$$
X_{a}^{L} \oplus X_{b}^{L} \oplus X_{c}^{L} \oplus X_{d}^{L}=0
$$

also holds with probability $2^{-6}$. Combining it with Equation (9) yields

$$
\operatorname{Pr}\left[\left(X_{c} \oplus X_{d}=\beta\right) \mid\left(X_{a} \oplus X_{b}=\beta\right) \wedge\left(Y_{a} \oplus Y_{c}=\gamma\right) \wedge\left(Y_{b} \oplus Y_{d}=\gamma\right)\right]=2^{-6}
$$

Therefore, the overall probability of the related-key sandwich distinguisher is

$$
(1 / 4)^{2} \cdot(1 / 4)^{2} \cdot 2^{-6}=2^{-14},
$$

which is much higher than the probability of $(1 / 4)^{2} \cdot(1 / 4)^{2} \cdot 2^{-32}=2^{-40}$ which is expected by the naive analysis of the sandwich structure.

\subsection{Experimental Verification}

To verify the properties of the new distinguisher, we used the official code available as an appendix in 24]. The verification experiment was set up as follows: In each test we randomly chose a key quartet satisfying the required key differences. We then generated $2^{16}$ quartets by following the boomerang procedure described above. We utilized a slight improvement of the first differential suggested in [13] that increases its probability in the encryption direction by a factor of 2 by fixing the value of two plaintext bits. Hence, we expect the number of right quartets in each test to be distributed according to a Poisson distribution with a mean value of $2^{16} \cdot 2^{-14} \cdot 2=8$. We repeated the test 100,000 times, and obtained a distribution which is extremely close to the expected distribution. The full results are summarized in Table 3 .

${ }^{1}$ This estimate is based on a randomness assumption that could be inaccurate in our case due to dependence between the differential characteristics. However, the experiments presented below verify that this probability is indeed as expected. 


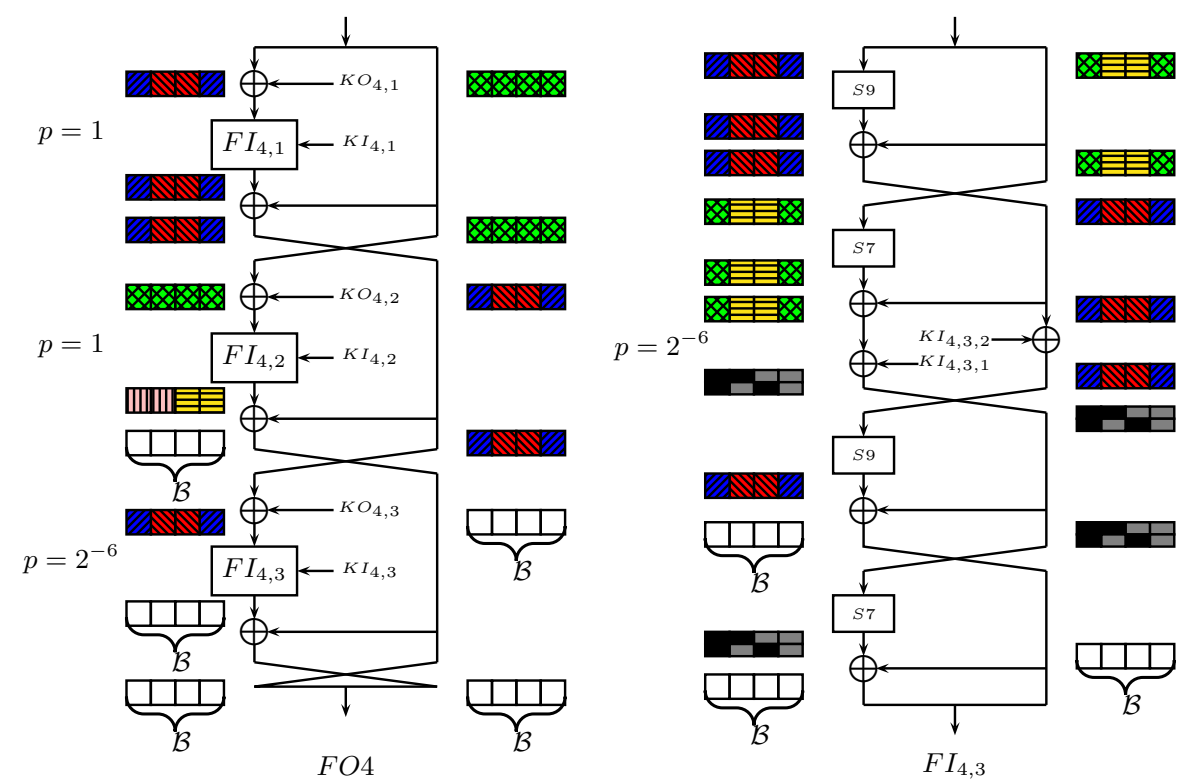

Values marked by the same color and style are equal. Values marked by $\mathcal{B}$ are balanced (i.e., the XOR of all four values is 0 ). The values in $F I_{4,3}$ which are either smooth gray or smooth black suggest one of two possible cases.

Fig. 5. The Development of Differences in $\mathrm{FO}_{4}$ and in $\mathrm{FI}_{4,3}$

\section{Related-Key Sandwich Attack on the Full KASUMI}

Our attack on the full KASUMI (depicted in Figure 6) applies the distinguisher presented in Section 4 to rounds 1-7, and retrieves subkey material in round 8 . Let $\Delta K_{a b}=\left(0,0,8000_{x}, 0,0,0,0,0\right)$ and $\Delta K_{a c}=\left(0,0,0,0,0,0,8000_{x}, 0\right)$, and let $K_{a}, K_{b}=K_{a} \oplus \Delta K_{a b}, K_{c}=K_{a} \oplus \Delta K_{a c}$, and $K_{d}=K_{c} \oplus \Delta K_{a b}$ be the unknown related keys we wish to retrieve.

The attack algorithm is as follows:

\section{Data Collection Phase:}

(a) Choose a structure of $2^{24}$ ciphertexts of the form $C_{a}=\left(X_{a}, A\right)$, where $A$ is fixed and $X_{a}$ assumes $2^{24}$ arbitrary different values. Ask for the decryption of all the ciphertexts under the key $K_{a}$ and denote the plaintext corresponding to $C_{a}$ by $P_{a}$. For each $P_{a}$, ask for the encryption of $P_{b}=P_{a} \oplus\left(0_{x}, 00100000_{x}\right)$ under the key $K_{b}$ and denote the resulting ciphertext by $C_{b}$. Store the pairs $\left(C_{a}, C_{b}\right)$ in a hash table indexed by the 32 -bit value $C_{b}^{R}$ (i.e., the right half of $C_{b}$ ).

(b) Choose a structure of $2^{24}$ ciphertexts of the form $C_{c}=\left(Y_{c}, A \oplus 00100000_{x}\right)$, where $A$ is the same constant as before, and $Y_{c}$ assumes $2^{24}$ arbitrary different values. Ask for the decryption of the ciphertexts under the key $K_{c}$ and denote the plaintext corresponding to $C_{c}$ by $P_{c}$. For each $P_{c}$, ask for the encryption of $P_{d}=P_{c} \oplus\left(0_{x}, 00100000_{x}\right)$ under the key $K_{d}$ and denote 
the resulting ciphertext by $C_{d}$. Then, access the hash table in the entry corresponding to the value $C_{d}^{R} \oplus 00100000_{x}$, and for each pair $\left(C_{a}, C_{b}\right)$ found in this entry, apply Step 2 on the quartet $\left(C_{a}, C_{b}, C_{c}, C_{d}\right)$.

In the first step described above, the $\left(2^{24}\right)^{2}=2^{48}$ possible quartets are filtered according to a condition on the 32 difference bits which are known (due to the output difference $\delta$ of the distinguisher), which leaves about $2^{16}$ quartets with the required differences.

In Step 2 we can identify the right quartets instantly using an extremely lucky property of the KASUMI structure. We note that a pair $\left(C_{a}, C_{c}\right)$ can be a right quartet if and only if

$$
C_{a}^{L} \oplus F L 8\left(F O 8\left(C_{a}^{R}\right)\right)=C_{c}^{L} \oplus F L 8\left(F O 8\left(C_{c}^{R}\right)\right),
$$

since by the Feistel structure, this is the only case of which the difference after round 7 is the output difference of the sandwich distinguisher (i.e., $\delta=$ $\left.\left(0_{x}, 00100000_{x}\right)\right)$. However, the values $C_{a}^{R}$ and $C_{c}^{R}$ are fixed for all the considered ciphertexts, and hence Equation (22) yields

$$
C_{a}^{L} \oplus C_{c}^{L}=F L 8(F O 8(A)) \oplus F L 8\left(F O 8\left(A \oplus 00100000_{x}\right)\right)=\text { const. }
$$

Thus, the value $C_{a}^{L} \oplus C_{c}^{L}$ is equal for all the right quartets. This allows us to perform the following simple filtering:

\section{Identifying the Right Quartets:}

(a) Insert the approximately $2^{16}$ remaining quartets $\left(C_{a}, C_{b}, C_{c}, C_{d}\right)$ into a hash table indexed by the 32 -bit value $C_{a}^{L} \oplus C_{c}^{L}$, and apply Step 3 only to bins which contain at least three quartets.

Since the probability of a 3 -collision in a list of $2^{16}$ random 32-bit values is lower than $\left(\begin{array}{c}2^{16} \\ 3\end{array}\right) \cdot 2^{-64} \leq 2^{-18}$, with very high probability only the right quartets remain after this filtering.

In the following step, we treat all the remaining quartets as right quartets. Under this assumption, we know not only the actual inputs to round 8, but also the differences in the outputs of round 8 .

\section{Analyzing Right Quartets:}

(a) For each remaining quartet $\left(C_{a}, C_{b}, C_{c}, C_{d}\right)$, guess the 32 -bit value of $K O_{8,1}$ and $K I_{8,1}$. For the two pairs $\left(C_{a}, C_{c}\right)$ and $\left(C_{b}, C_{d}\right)$ use the value of the guessed key to compute the input and output differences of the OR operation in the last round of both pairs. For each bit of this 16bit OR operation of $F L 8$, the possible values of the corresponding bit of $K L_{8,2}$ are given in Table 2. On average $(8 / 16)^{16}=2^{-16}$ values of $K L_{8,2}$ are suggested by each quartet and guess of $K O_{8,1}$ and $K I_{8,1} 2$ Since all the right quartets suggest the same key, all the wrong keys are discarded with overwhelming probability, and the attacker obtains the correct value of $\left(K O_{8,1}, K I_{8,1}, K L_{8,2}\right)$.

$\overline{2}$ The simple proof of this claim is given in Section 4.3 of 8 . 
Table 2. Possible Values of $K L_{8,2}$ and $K L_{8,1}$

OR $-K L_{8,2}$
\begin{tabular}{|c|cccc|}
\hline \multirow{2}{*}{$\left(X_{a c}^{\prime}, Y_{a c}^{\prime}\right)$} & \multicolumn{4}{c|}{$\left(X_{b d}^{\prime}, Y_{b d}^{\prime}\right)$} \\
\hline$(0,0)$ & $(0,0)$ & $(0,1)$ & $(1,0)$ & $(1,1)$ \\
$(0,1)$ & $-0,1\}$ & - & 1 & 0 \\
$(1,0)$ & 1 & - & - & - \\
$(1,1)$ & 0 & - & - & 0 \\
\hline
\end{tabular}

\begin{tabular}{|c|cccc|}
\multicolumn{4}{c}{ AND $-K L_{8,1}$} \\
\hline \multirow{2}{*}{$\left(X_{a c}^{\prime}, Y_{a c}^{\prime}\right)$} & \multicolumn{4}{c|}{$\left(X_{b d}^{\prime}, Y_{b d}^{\prime}\right)$} \\
\hline$(0,0)$ & $(0,0)$ & $(0,1)$ & $(1,0)$ & $(1,1)$ \\
$(0,1)$ & $-1\}$ & - & 0 & 1 \\
$(1,0)$ & 0 & - & - & - \\
$(1,1)$ & 1 & - & - & - \\
\hline
\end{tabular}

* The two bits of the differences are denoted by (input difference, output difference): $\left(X_{1}^{\prime}, Y_{1}^{\prime}\right)$ for one pair and $\left(X_{2}^{\prime}, Y_{2}^{\prime}\right)$ for the other pair.

Table 3. The Number of Right Quartets in 100,000 Experiments

\begin{tabular}{lccccccccc}
\hline Right Quartets & 0 & 1 & 2 & 3 & 4 & 5 & 6 & 7 & 8 \\
\hline Theory (Poi $(8))$ & 34 & 268 & 1,073 & 2,863 & 5,725 & 9,160 & 12,214 & 13,959 & 13,959 \\
\hline Experiment & 32 & 259 & 1,094 & 2,861 & 5,773 & 9,166 & 12,407 & 13,960 & 13,956 \\
\hline \hline Right Quartets & 9 & 10 & 11 & 12 & 13 & 14 & 15 & 16 & 17 \\
\hline Theory (Poi(8)) & 12,408 & 9,926 & 7,219 & 4,813 & 2,962 & 1,692 & 903 & 451 & 212 \\
Experiment & 12,230 & 9,839 & 7,218 & 4,804 & 3,023 & 1,672 & 859 & 472 & 219 \\
\hline \hline Right Quartets & 18 & 19 & 20 & 21 & 22 & 23 & 24 & 25 & \\
\hline Theory (Poi $(8))$ & 94 & 40 & 16 & 6 & 2 & 0.8 & 0.26 & 0.082 & \\
\hline Experiment & 89 & 39 & 13 & 12 & 2 & 0 & 0 & 1 & \\
\hline
\end{tabular}

(b) Guess the 32-bit value of $K O_{8,3}$ and $K I_{8,3}$, and use this information to compute the input and output differences of the AND operation in both pairs of each quartet. For each bit of the 16-bit AND operation of $F L 8$, the possible values of the corresponding bit of $K L_{8,1}$ are given in Table 2. On average $(8 / 16)^{16}=2^{-16}$ values of $K L_{8,1}$ are suggested by each quartet and guess of $K O_{8,3}, K I_{8,3}$, and thus the attacker obtains the correct value of $\left(K O_{8,3}, K I_{8,3}, K L_{8,1}\right)$.

4. Finding the Right Key: For each value of the 96 bits of $\left(K O_{8,1}, K I_{8,1}\right.$, $\left.K O_{8,3}, K I_{8,3}, K L_{8,1}, K L_{8,2}\right)$ suggested in Step 3, guess the remaining 32 bits of the key, and perform a trial encryption.

The data complexity of the attack is $2^{25}$ chosen ciphertexts and $2^{25}$ adaptively chosen plaintexts encrypted/decrypted under one of four keys. The time complexity is dominated by the trial encryptions performed in step 4 to find the last 32 bits of the key, and thus it is approximately equal to $2^{32}$ encryptions. The probability of success is approximately $76 \%$ (this is the probability of having at least three right pairs in the data pool).

The memory complexity of the attack is also very moderate. We just need to store $2^{26}$ plaintext/ciphertext pairs, where each pair takes 16 bytes. Hence, the total amount of memory used in the attack is $2^{30}$ bytes, i.e., 1 GByte of memory. 
Table 4. The Number of Identified Right Quartets in 1,000 tests

\begin{tabular}{lccccccccccc}
\hline Right Quartets & $0 / 1 / 2$ & 3 & 4 & 5 & 6 & 7 & 8 & 9 & 10 & 11 & 12 \\
\hline Theory $(\operatorname{Poi}(4))$ & 238 & 195 & 195 & 156 & 104 & 60 & 30 & 13 & 5 & 2 & 0.6 \\
\hline Experiment & 247 & 197 & 180 & 167 & 112 & 52 & 30 & 7 & 4 & 3 & 1 \\
\hline
\end{tabular}

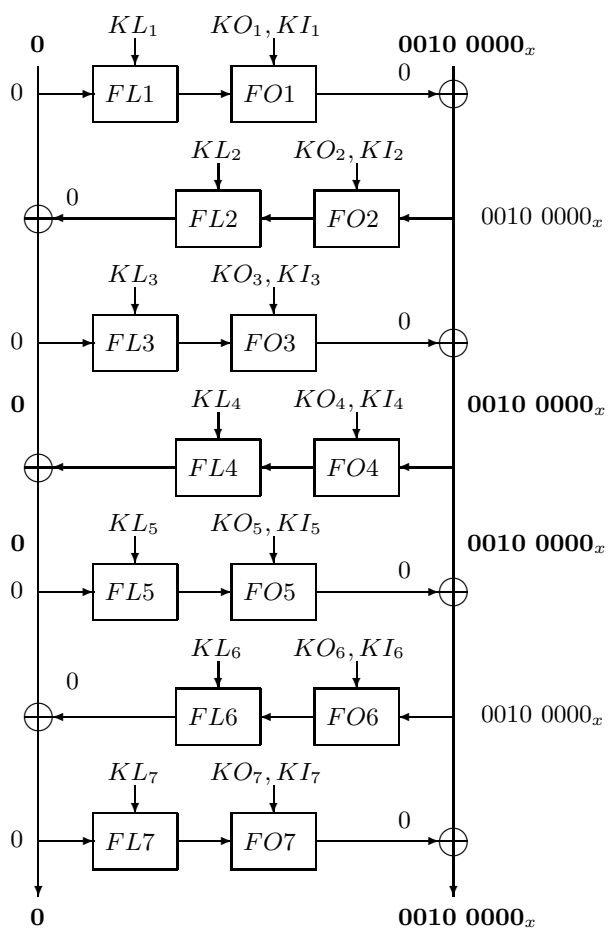

Fig. 6. The 7-Round Related-Key Sandwich Distinguisher of KASUMI

\subsection{Experimental Verification}

We performed two types of experiments to verify our attack. In the first experiment, we just generated the required data, and located the right quartets (thus verifying the correctness of our randomness assumptions). The second experiment was the application of the full attack (both with and without the final exhaustive search over the remaining 32 key bits). All our experiments were carried out on an Intel Core Duo 2 machine with a T7200 CPU (2 GHz, 4 MB L2 Cache, 2 GB RAM, Linux-2.6.27 kernel, with gcc 4.3.2 and standard optimization flags (-03, -fomit-frame-pointers, -funroll-loops), single core, single thread).

The first experiment was conducted 1,000 times. In each test, we generated the data and found candidate quartets according to Steps 1 and 2 of the 
attack algorithm. Once these were found, we partially decrypted the quartets, and checked how many quartets were right ones. Table 4 details the outcome of these experiments, which follow the expected distribution.

The second experiment simulated the full attack. We repeated it 100 times, and counted in each case how many times the final exhaustive search over $2^{32}$ possible keys would have been evoked. In 78 out of these 100 experiments, the key was found when 3 or more quartets were identified to be right ones (the expected number was 76.1).

About $50 \%$ of the tests were able to identify the right key by invoking either 2 or 4 exhaustive searches. As the first part of the attack (which identifies candidate quartets) takes about 8 minutes, and each exhaustive search (using the official KASUMI source code) takes about 26 minutes, we could find the full 128 bit key in about $50 \%$ of our tests in less than 112 minutes (using a single core). It is important to note that by increasing the running time, one can increase the success rate of the attack without increasing its data requirements.

\section{Summary}

In this paper we develop a new sandwich attack on iterated block ciphers, and use it to reduce the time complexity of the best known attack on the full KASUMI from an impractical $2^{76}$ to the very practical $2^{32}$. However, the new attack uses both related keys and chosen messages, and thus it might not be applicable to the specific way in which KASUMI is used as the A5/3 encryption algorithm in third generation GSM telephony. Our main point was to show that contrary to the assurances of its designers, the transition from MISTY to KASUMI led to a much weaker cryptosystem, which should be avoided in any application in which related-key attacks can be mounted.

\section{References}

1. A5/1 Security Project, Creating A5/1 Rainbow Tables (2009), http://reflextor.com/trac/a51

2. Barkan, E., Biham, E.: Conditional Estimators: an Effective Attack on A5/1. In: Preneel, B., Tavares, S. (eds.) SAC 2005. LNCS, vol. 3897, pp. 1-19. Springer, Heidelberg (2006)

3. Barkan, E., Biham, E., Keller, N.: Instant Ciphertext-Only Cryptanalysis of GSM Encrypted Communication. In: Boneh, D. (ed.) CRYPTO 2003. LNCS, vol. 2729, pp. 600-616. Springer, Heidelberg (2003)

4. Biham, E.: New Types of Cryptanalytic Attacks Using Related Keys. Journal of Cryptology 7(4), 229-246 (1994)

5. Biham, E., Dunkelman, O., Keller, N.: The Rectangle Attack - Rectangling the Serpent. In: Pfitzmann, B. (ed.) EUROCRYPT 2001. LNCS, vol. 2045, pp. 340357. Springer, Heidelberg (2001)

6. Biham, E., Dunkelman, O., Keller, N.: New Results on Boomerang and Rectangle Attacks. In: Daemen, J., Rijmen, V. (eds.) FSE 2002. LNCS, vol. 2365, pp. 1-16. Springer, Heidelberg (2002) 
7. Biham, E., Dunkelman, O., Keller, N.: Related-Key Boomerang and Rectangle Attacks. In: Cramer, R. (ed.) EUROCRYPT 2005. LNCS, vol. 3494, pp. 507-525. Springer, Heidelberg (2005)

8. Biham, E., Dunkelman, O., Keller, N.: A Related-Key Rectangle Attack on the Full KASUMI. In: Roy, B. (ed.) ASIACRYPT 2005. LNCS, vol. 3788, pp. 443-461. Springer, Heidelberg (2005)

9. Biryukov, A.: The Boomerang Attack on 5 and 6-Round Reduced AES. In: Dobbertin, H., Rijmen, V., Sowa, A. (eds.) AES 2005. LNCS, vol. 3373, pp. 11-15. Springer, Heidelberg (2005)

10. Biryukov, A., De Cannière, C., Dellkrantz, G.: Cryptanalysis of SAFER++. In: Boneh, D. (ed.) CRYPTO 2003. LNCS, vol. 2729, pp. 195-211. Springer, Heidelberg (2003)

11. Biryukov, A., Khovratovich, D.: Related-key Cryptanalysis of the Full AES-192 and AES-256. In: Matsui, M. (ed.) ASIACRYPT 2009. LNCS, vol. 5912, pp. 1-18. Springer, Heidelberg (2009)

12. Biryukov, A., Shamir, A., Wagner, D.: Real Time Cryptanalysis of A5/1 on a PC. In: Schneier, B. (ed.) FSE 2000. LNCS, vol. 1978, pp. 1-18. Springer, Heidelberg (2001)

13. Blunden, M., Escott, A.: Related Key Attacks on Reduced Round KASUMI. In: Matsui, M. (ed.) FSE 2001. LNCS, vol. 2355, pp. 277-285. Springer, Heidelberg (2002)

14. Briceno, M., Goldberg, I., Wagner, D.: A Pedagogical Implementation of the GSM A5/1 and A5/2 "voice privacy" encryption algorithms (1999), http://cryptome.org/gsm-a512.htm

15. Dunkelman, O., Keller, N.: An Improved Impossible Differential Attack on MISTY1. In: Pieprzyk, J. (ed.) ASIACRYPT 2008. LNCS, vol. 5350, pp. 441-454. Springer, Heidelberg (2008)

16. Ekdahl, P., Johansson, T.: Another Attack on A5/1. IEEE Transactions on Information Theory 49(1), 284-289 (2003)

17. Golic, J.D.: Cryptanalysis of Alleged A5 Stream Cipher. In: Fumy, W. (ed.) EUROCRYPT 1997. LNCS, vol. 1233, pp. 239-255. Springer, Heidelberg (1997)

18. Hong, S., Kim, J., Kim, G., Lee, S., Preneel, B.: Related-Key Rectangle Attacks on Reduced Versions of SHACAL-1 and AES-192. In: Gilbert, H., Handschuh, H. (eds.) FSE 2005. LNCS, vol. 3557, pp. 368-383. Springer, Heidelberg (2005)

19. Kelsey, J., Schneier, B., Wagner, D.: Key Schedule Cryptanalysis of IDEA, G-DES, GOST, SAFER, and Triple-DES. In: Koblitz, N. (ed.) CRYPTO 1996. LNCS, vol. 1109, pp. 237-251. Springer, Heidelberg (1996)

20. Kim, J., Kim, G., Hong, S., Hong, D.: The Related-Key Rectangle Attack - Application to SHACAL-1. In: Wang, H., Pieprzyk, J., Varadharajan, V. (eds.) ACISP 2004. LNCS, vol. 3108, pp. 123-136. Springer, Heidelberg (2004)

21. Kim, J., Hong, S., Preneel, B., Biham, E., Dunkelman, O., Keller, N.: Related-Key Boomerang and Rectangle Attacks, IACR ePrint report 2010/019

22. Matsui, M.: Block encryption algorithm MISTY. In: FSE 1997. LNCS, vol. 1267, pp. 64-74. Springer, Heidelberg (1997)

23. Murphy, S.: The Return of the Boomerang, technical report RHUL-MA-2009-20, Department of Mathematics, Royal Holloway, University of London (2009), http://www.rhul.ac.uk/mathematics/techreports 
24. 3rd Generation Partnership Project, Technical Specification Group Services and System Aspects, 3G Security, Specification of the 3GPP Confidentiality and Integrity Algorithms; Document 2: KASUMI Specification, V3.1.1 (2001)

25. 3rd Generation Partnership Project, Technical Specification Group Services and System Aspects, 3G Security, Specification of the A5/3 Encryption Algorithms for GSM and ECSD, and the GEA3 Encryption Algorithm for GPRS; Document 4: Design and evaluation report, V6.1.0 (2002)

26. TECHNEWSWORLD, Hackers Jimmy GSM Cellphone Encryption (published 29/12/2009), http://www.technewsworld.com/rsstory/68997.html

27. Wagner, D.: The Boomerang Attack. In: Knudsen, L.R. (ed.) FSE 1999. LNCS, vol. 1636, pp. 156-170. Springer, Heidelberg (1999) 